\title{
La educación social del alumnado con sobredotación y una propuesta para mejorarla: la tutoría entre iguales
}

Plaza-de-la-Hoz, Jesús

La educación social del alumnado con sobredotación y una propuesta para mejorarla: la tutoría entre iguales

Revista Educación, vol. 43, núm. 2, 2019

Universidad de Costa Rica, Costa Rica

Disponible en: http://www.redalyc.org/articulo.oa?id=44058158008

DOI: https://doi.org/10.15517/revedu.v43i2.30792

Esta obra está bajo una Licencia Creative Commons Atribución-NoComercial-SinDerivar 3.0 Internacional. 


\title{
La educación social del alumnado con sobredotación y una propuesta para mejorarla: la tutoría entre iguales
}

\author{
Jesús Plaza-de-la-Hoz \\ Universidad Internacional de La Rioja, España \\ jesus.plaza@unir.net \\ iD http://orcid.org/0000-0002-6966-4214
}

Encouraging Social Education for Gifted students: Peer Tutoring

DOI: https://doi.org/10.15517/revedu.v43i2.30792

Redalyc: http://www.redalyc.org/articulo.oa?id=44058158008

Recepción: 05 Octubre 2017

Aprobación: 03 Mayo 2019

\section{RESUMEN:}

Una sociedad sostenible plantea retos a las generaciones futuras, y al sistema educativo. Nuestro mundo tecnológico globalizado requiere que todos los talentos, especialmente los sobredotados, trabajen unidos para construir una sociedad justa y equilibrada. Pero ¿̇cómo fomentar su responsabilidad? Este estudio teórico sobre la noción del talento y sus distintos modelos recogidos en la literatura, presta especial atención a las inteligencias múltiples y a los aspectos socioemocionales; se plantea desde la educación social y el aprendizaje como servicio. Finalmente, reinterpreta la tutoría entre iguales como un modo de fomentar la responsabilidad social en el alumnado. Para la alta capacidad especialmente, puede ser un buen entrenamiento para ejercer un liderazgo proactivo, solidario y democrático, al servicio de las comunidades de referencia.

Palabras Clave: Desarrollo del talento, Aprendizaje del servicio, Educación social, Aprendizaje entre iguales, Mente ética.

\section{Abstract:}

A sustainable society poses challenges to future generations as well as the educational system. Our globalized technological world requires all sorts of talents. Under this premise, it is important for all individuals, particularly the gifted, to work together to help build a fair and balanced society. But how do we encourage responsibility? This theoretical study addresses the concept of talent and its different models as it is reviewed in literature. It particularly emphasizes Social Education and Learning as it pertains to service. Finally, it reinterprets peer tutoring as a way of encouraging social responsibility among the student body. This may be excellent training, especially for high achievers, to practice proactive, supportive and democratic leadership in the service of concerned communities.

KEYWORDS: Talent Development, Service Learning, Social Education, Peer Teaching, Ethical Mindset.

\section{INTRODUCCIÓN}

El objeto de este estudio es reflexionar sobre la sobredotación y su papel en la mejora de la educación social del alumnado. Nuestro mundo globalmente interconectado requiere respuestas por parte del sistema educativo. Los nuevos retos se manifiestan en las aulas, cuya pluralidad incluye la presencia multicultural y la diversidad de capacidades y talentos. Además, el futuro próximo debe aprovechar las energías de las nuevas generaciones para hacerse cargo del contexto emergente. En esta línea, descubrir y alentar las inteligencias sobresalientes lo exige tanto la justicia como la equidad del sistema. A la finalidad que Renzulli señala al desarrollo del talento ("the individual self-fulfillment and contributing to the reservoir of people who will become the scientists, artists, the philosophers, the business people, politicians, the leaders of our world" [Hernández y Gulsah, 2014, p. 9]). se le podría dotar de un enfoque social-solidario, en línea con la red de redes en la que se forja la sociedad del tercer milenio. Si orientamos así el cuidado de los sobredotados, el resultado esperanzador será una promoción solidaria del talento y una mejora de todos para una sociedad equilibrada y próspera. De hecho, recientes trabajos de Renzulli apuntan en esta dirección (Renzulli, 2012; Renzulli y D’Souza, 2014; Sternberg, 2018). 
Una cuestión asociada es la visión competitiva de la perspectiva de mercado, la cual afecta a países, compañías e individuos (trabajadores y estudiantes): se trata de la creencia de que un resultado de suma positiva solo se alcanza por medio de juegos de suma cero, y que el triunfo de cada cual redundará en el beneficio de todos. En este artículo se parte de la posición opuesta, con la convicción de que hacen falta modelos alternativos de excelencia social y personal (Pérez, 2005). Solo si en origen la educación y socialización de los jóvenes siembra cooperación y orientación al grupo, cabrá construir una sociedad más solidaria y justa; y a la vez, lograr el éxito de cada uno como persona. El camino consistirá en poner desde un principio el talento individual al servicio del conjunto.

Pero como el talento está por naturaleza desigualmente distribuido (Tourón, 2010), el sistema escolar deberá educar ese talento socialmente, para que no se malogre por una mal entendida apuesta de mínimos, ni se distancie de sus iguales (Plucker y Callahan, 2014) en una carrera por la excelencia individual, tantas veces centrada en parámetros parciales como el puramente intelectual.

En este artículo se plantea cómo educar al alumnado sobredotado en el servicio a sus iguales en el entorno escolar. Analiza el concepto de talento usual en la literatura, y las diferentes concepciones de la inteligencia sobresaliente. Reflexiona sobre aspectos socioemocionales en comparación con estudios de casos sobre la necesidad de fomentar las altas capacidades en beneficio propio y colectivo. Propone la reinterpretación de la tutoría entre iguales con múltiples cauces de implementación práctica. La necesidad de aplicarla caso por caso supone el principal reto y la mayor limitación.

\section{Un problema futuro y actual}

El presente contexto de la globalización plantea dos retos. De una parte, cómo incrementar los índices de desarrollo en beneficio del planeta. Un crecimiento diferencial se hace cada vez más insostenible, toda vez que los cambios provocan transformaciones múltiples y compartidas, tanto en el bienestar de quienes se benefician, como en el malestar de la gente excluida. Las desigualdades flagrantes generan movimientos de rechazo con resultados imprevisibles de consecuencias globales.

Otro problema asociado es la necesidad de coordinar medidas de alcance nacional en un contexto político y económico supranacional. En el proceso puede perderse el capital social que sostiene nuestras democracias. Si las decisiones que nos afectan las toman expertos técnicos según necesidades de nivel macroeconómico, el resultado es la desafección política de las nuevas generaciones. En países como EUU se percibe una "profunda falta de empoderamiento cívico" (Levinson, 2012, p. 31), una pérdida y disminución de compromiso con la comunidad. Y no será suficiente con que algunas personas jóvenes se involucren personalmente en asociaciones de voluntariado de iniciativa privada; hace falta incrementar la responsabilidad social y la vinculación cívica de los jóvenes.

Las tecnologías pueden resultar de gran ayuda (LeCompte y Blevins, 2013; West, 2012), pero son instrumentales. Facilitarán el cambio de orientación hacia el protagonismo del alumno en la sociedad del conocimiento, si contemplan las características diferenciales del estudiantado. Para ello convendría implementar la formación docente (Llamas y Macías, 2016), y procurar una flexibilización del sistema (Tourón, 2010) que promueva que todos avancen, especialmente los sobredotados. La sociedad se beneficiará de la dedicación que hoy podamos ofrecer a nuestro alumnado. Y esa responsabilidad social futura al servicio del desarrollo de todos requiere fomentar en la formación de los sobredotados una conciencia global adecuadamente nutrida por la inteligencia emocional: pensamiento crítico, dinamismo para resolver problemas, preocupación y compasión por el bien común, conciencia de la interdependencia global, espíritu humanista, ciudadanía activa, aprendizaje del servicio (Van der Westhuizen y Maree, 2010). 


\section{DisTINTOS MODELOS SOBRE SUPERDOTACIÓN}

Resulta complejo definir la sobredotación y determinar la objetividad de diversos modelos. A veces se distingue superdotación (atributo casi físico que permite obtener una elevada puntuación en capacidad intelectual medida por un test y reflejada en un cociente) de alta capacidad (un ritmo de aprendizaje más amplio, creativo y profundo que el esperable para la edad considerada). En este artículo extendemos la sobredotación más allá de ese estrecho traje, en línea con expertos en altas capacidades (Gagné, 2015; Olzewski-Kubilius, Subotnik y Worrell, 2015; Pfeiffer, 2015; Tourón y Tourón, 2016; etc.); entendiendo que estas pueden desarrollar diversas dimensiones, una vez detectadas.

En resultados de investigaciones empíricas (Plucker y Callahan, 2014) se observa un solapamiento e interacción entre características cognitivas, afectivas y motivacionales. Si pensamos en los líderes ideales para la sociedad del futuro, cabe plantearse educar de modo cualitativamente diverso al alumnado sobredotado; en otras palabras, trasladar el capital intelectual y creativo al futuro capital social, funcional y ejecutivo. Para ello se debiera enfocar la formación temprana hacia la implicación directa, más que a una aséptica enseñanza-aprendizaje. El resultado podrían ser líderes sensibles a lo humano, al cuidado del medioambiente, a la preocupación por la participación democrática (Renzulli, 2012).

Pero el talento no es fijo ni homogéneo; se despliega en el tiempo con el apoyo conveniente y la inversión necesaria, con el esfuerzo y la elección personal para desarrollarlo en el área adecuada. Las inteligencias múltiples revelan distintas facetas del talento, respecto a dos componentes: el académico (inteligencia lingüística, lógico-matemática, naturalista y visoespacial), y el no académico (inteligencia corporal, musical y social) (Hernández, Ferrándiz, Ferrando, Prieto y Fernández, 2014). Cabe así considerar perfiles complejos y multidimensionales de alumnado superdotado, cuando presentan un rendimiento superior en algún dominio específico, además de una habilidad potencial general. Para procurarles una atención individualizada, enriqueciendo el currículum y adaptándolo al alumno, dentro de un modelo escolar inclusivo, cabe dirigir esas habilidades a proyectos personales y sociales.

El modelo de inteligencia exitosa (Sternberg et ál., 2010) propone el equilibrio entre las inteligencias analítica cognitiva, sintética o creativa, y práctica adaptada al entorno. Un equilibrio que presta atención especial a los sobredotados, pero que también beneficia al resto del grupo al rentabilizar los puntos fuertes compensando los diversos tipos de habilidades en cada campo.

Renzulli por su parte define el Modelo de Enriquecimiento Escolar. Su propuesta adopta una postura proactiva frente a la estrategia de la educación de remedios; pues esta última propende a modelos de mínimo esfuerzo los cuales debilitan la capacidad del reto. Para que la escuela desarrolle el talento de todos debe incidir en las actividades sobresalientes de cada alumno, no solo en su inteligencia. Los alumnos y alumnas responden a un aprendizaje enriquecedor. El modelo reúne experiencias positivas ya experimentadas: centrarse más en el aprendizaje que en las destrezas, configurar currículos interdisciplinarios, evaluar el desempeño, añadir investigación y aplicación a las tareas tradicionales, etc. Si ponemos al alumno en el centro (sabiendo de dónde parte, cómo aprende, qué le interesa, en qué destaca), flexibilizamos los horarios (con algunas clases selectivas mensuales según áreas), y contamos con madres, padres, profesores, e incluso estudiantes mayores, para aportar cambios, se hace posible un consenso que no segregue, que atienda especialmente al alumnado en riesgo, y a la vez motive a todos.

Los aportes previos, de Sternberg y Renzulli, fueron adoptados por Aljughaiman (2010) para diseñar su Modelo de Enriquecimiento Oasis, al que añade las virtualidades del constructivismo. Especialmente centrado en estudiantes con sobredotación, el modelo propone unificar las áreas de investigación, pensamiento y afectividad para elaborar proyectos y presentarlos al final de cada período de aprendizaje. $\mathrm{Su}$ desarrollo recorre tres etapas: exploración, perfeccionamiento y creatividad. En el tercer nivel de enriquecimiento, se incluyen habilidades sociales relacionadas con el futuro y los problemas de la comunidad. De igual modo considera que el desarrollo de rasgos personales y socioafectivos ayudará a rentabilizar mejor 
las habilidades cognitivas, al generar responsabilidad individual y social. El resultado es una alta motivación y una mejora de todo el claustro.

\section{EL TALENTO SOCIAL A LA LUZ DE LA TEORÍA DE LAS INTELIGENCIAS MÚLTIPLES}

La diferenciación de Gardner acerca de múltiples dimensiones de la inteligencia ha generado un campo de estudio en el área de las altas capacidades. Como se indicó previamente, la superdotación ha de tener en cuenta dos tipos de componentes: el académico, con sus capacidades lingüística, lógica, matemática, espacial y natural; y el no académico, que englobaría las inteligencias corporal, musical y social. Ello añade complejidad a la caracterización del superdotado, pues la alta capacidad general se distingue de los específicos y diferentes tipos de talentos. Al diferir sus motivaciones e intereses intelectuales, el superdotado puede aburrirse si no se le proporciona trabajo de complejidad adecuada a su desarrollo, o bien puede ser rechazado por los amigos si no se potencia una socialización adecuada. Esta complejidad requiere el concurso de padres, profesores y compañeros, tanto dentro como fuera del aula.

Atendiendo a lo que Gardner $(2008,21)$ denomina ethical mind, la experiencia de que el alumnado con talentos especiales suele alcanzar posiciones sociales más reconocidas, sobre todo por sus ingresos económicos, permite ilustrar la importancia de educar en esta área específica de la inteligencia, que podríamos conectar en general con las habilidades sociales proactivas, enriqueciendo así al alumnado sobredotado y al no sobredotado en el futuro. El desarrollo de una mente ética sería por tanto uno de los principales objetivos de la educación en general y de la de los sobredotados en particular. Por este citado autor entiende la capacidad de reconocer papeles y responsabilidades personales de cada uno como miembro de las comunidades de pertenencia, de modo que entienda las necesidades y deseos de la sociedad en la que vive y actúe para mejorarla por encima de su interés particular.

Considerando las variadas capacidades del estudiantado con talento, la formación en un pensamiento ético podrá modularse, con razonamientos solidarios centrados en el desarrollo de la propia identidad -sobre todo en la adolescencia-, o con actividades prácticas de voluntariado que enfrenten al alumnado con problemas sociales de su entorno.

Además de tipos de inteligencia, en la realidad se dan perfiles de talento complejos, donde varias capacidades por encima de la media cooperan para dotar de excelencia al que las posee. Por ejemplo, los talentos académicos reúnen habilidades de lenguaje, análisis lógico y memoria. A ello se une la variabilidad de estilos de enseñanza y de aprendizaje, que habrán de adaptarse al perfil de cada estudiante superdotado. Entre las características personales que más pueden influir en el profesor de estos alumnos y alumnas, algunos autores destacan la creatividad (Gallagher y Gallagher, 1994); y entre los recursos instruccionales, la individualización enriquece o compacta el currículum facilitando el estudio independiente guiado por tutorías adecuadas.

Centrándonos en el talento social, Castelló (2002) lo define como la capacidad de ayudar al grupo en sus objetivos mejorando las relaciones humanas, así como codificar y tomar decisiones sobre información del medio social. Sería, por tanto, una especial habilidad de interacción. Gardner distingue entre la inteligencia intrapersonal (conocimiento de sí, autorreflexión, sentimientos, metacognición y autopercepción) y la interpersonal (relación con los otros). Pues bien, la intervención más eficaz y productiva con estudiantes que destacan en talento social sería que usen sus recursos sociales para fomentar el trabajo cooperativo y su mediación para facilitar la integración de otros en el grupo (Prieto, 2004). Por eso es pertinente preguntarse si el estudiantado talentoso goza también de habilidades sociales. 


\section{LA INTELIGENCIA SOCIOEMOCIONAL ENTRE EL ALUMNADO SOBREDOTADO}

Apoyado en los trabajos de Inkeles (1966) e Inkeles y Smith (1974), y en estudios empíricos más recientes de Cunha y Heckman (2010), Levin (2013) señala que las habituales clasificaciones de tipo cognitivo son insuficientes para mostrar el talento del alumnado; y que al presionar sobre las políticas educativas es probable que las metas solo cognitivas debiliten la productividad social y económica. De ahí la importancia de tener en cuenta las actitudes, comportamiento y valores en la formación: el esfuerzo, la autodisciplina, la cooperación, la autoproyección, la tolerancia o el respeto, entre otros. Estas capacidades son tan importantes como las intelectuales, incluso más a largo plazo, para crear sociedades productivas, equitativas, y socialmente cohesionadas (Gradstein y Justman, 2002).

Sin embargo, no es fácil medir habilidades no cognitivas. Entre otras razones porque la inteligencia social incluye múltiples dimensiones (López, 2007). Los superdotados tienen un nivel de razonamiento igual o superior al resto, aunque no está claro que sobresalgan en competencia emocional; resulta más significativa su capacidad teórica de resolver problemas sociales que de establecer estrategias adecuadas para solucionarlos. Porque no es lo mismo la conciencia, el pensamiento y aun la empatía, que la acción práctica. En esto último, el contexto y la experiencia influyen más que la inteligencia en general.

Además, las altas capacidades pueden influir negativamente en el equilibrio emocional de los superdotados si provocan rechazo de sus iguales. Por eso importa reconocer su normalidad psicológica (Peñas, 2008; López y Calero, 2018 y evitar estereotipos que pueden llevarles a ocultar voluntariamente sus habilidades, sobre todo en la adolescencia, y especialmente entre las chicas. Una detección precoz de las altas capacidades ayudará a que se acepten y sean aceptados con naturalidad, pues algunos estudios (Mohammadyari, 2013) correlacionan la autoconciencia emocional con el logro académico de los superdotados.

Otro estudio empírico (Mora, Mathiesen, Merino, Castro y Navarro, 2010) muestra que la responsabilidad social de los superdotados correlaciona solo con la nota media, mientras la conducta prosocial lo hace con el vocabulario. Podría ser que la inteligencia social se corresponda con la inteligencia en general, mientras las habilidades prácticas necesitan capacidades interactivas que se desarrollan según el contexto.

Por tanto, dentro de la normalidad psicológica del alumno especialmente dotado, sus altas capacidades cognitivas necesitan que otras habilidades no cognitivas sean tenidas en cuenta y fomentadas para implementar el desarrollo integral del estudiante.

\section{HABILIDADES SOCIALES Y SUPERDOTACIÓN}

Un aspecto concreto de las habilidades no cognitivas de tipo socioemocional, es el relacionado con el liderazgo. Aunque se diferencian muchos tipos, aquí se atiende al sentido positivo de conciencia social. Si es importante dedicar recursos al desarrollo de habilidades no cognitivas, en el mismo grado en que se dedican a la instrucción cognitiva, más importante aún es promover un incremento de capital social y facilitar experiencias prácticas que creen un efectivo y compasivo liderazgo en aquellos jóvenes con excepcionales capacidades de aprendizaje (Renzulli y D’Souza, 2014; Renzulli, Sands y Heilbronnor, 2018). Los aspectos relacionados con la inteligencia social interaccionan positivamente con el desarrollo cognitivo, pero a la vez juegan un importante papel en la formación de creencias, valores y actitudes que orientan la acción. Los alumnos y alumnas con alto potencial acabarán asumiendo posiciones de relevancia en la sociedad futura; de ahí que el tipo de liderazgo que ejerzan tenga un impacto especialmente positivo si se trata de un liderazgo moral, ético y compasivo al servicio de la mejora de la sociedad. Ejemplos en la historia del pasado siglo avalan la potencia destructiva de liderazgos de sentido opuesto. Por eso sería importante desarrollar capacidades solidarias en el alumnado, con especial relevancia en los sobredotados.

Una posibilidad para lograrlo es promover comunidades prosociales (Tyler, 2004), cuyos miembros se preocupan por el bienestar de los otros y de la comunidad tanto como de ellos mismos. Se trata de ponerles 
en situación de adquirir sentido de la propia dignidad, conciencia social y procedimientos de justicia social. Para que internalicen estos valores y puedan poner en práctica la disposición a contribuir, debe contarse con ellos, a través de actividades adaptadas según la edad, para que desarrollen autonomía y responsabilidad. De ahí que un modelo de participación colaborativa sea el mejor instrumento para construir este liderazgo.

\section{RITMOS DE APRENDIZAJE Y FLEXIBILIZACIÓN DE ITINERARIOS}

La opción de adelantar materias de aprendizaje específicas para estudiantes con alta capacidad en ellas, o incluso avanzar cursos completos, se considera efectiva según la investigación empírica (Assouline, Colangelo, Lupkowski-Shoplik, Lipscomb y Forstadt, 2009). Y parece tener en general efectos sociales positivos (Gallagher, 2004; Robinson, 2004; Colangelo, Assouline y Gross, 2004; Rogers, 2007); sobre todo si tenemos en cuenta al alumnado sobredotado de escasos recursos o procedentes de minorías étnicas, que carecen de educación suplementaria privada. Con las medidas de prudencia aconsejables, como la voluntariedad del candidato, y teniendo en cuenta ante todo su bienestar, la aceleración permite equiparar programas de enseñanza en el nivel de conocimientos, ritmo, intereses y habilidades de este estudiantado. Las supuestas consecuencias negativas socioemocionales parecen responder a mitos y creencias sin fundamento, no a conclusiones de investigación: así, algunos estudios (Valadez, Pérez y Beltrán, 2010) corroboran el equilibrio psicológico de este alumnado e, incluso, una superioridad en inteligencia emocional, independientemente de la medida que se utilice, y especialmente a favor de las chicas. En este sentido, elevar las expectativas de estos alumnos y alumnas facilita plantear retos apropiados, sobre todo en regiones con el nivel educativo general todavía bajo, como Latinoamérica (Blumen, 2008, 2013), donde la identificación del estudiantado con talento y el tratamiento adecuado a sus circunstancias se muestra urgente. La aceleración podría ser una de las medidas para implementar, junto con enriquecer los programas educativos, ofrecer homeschooling tutorizado, o desarrollar aprendizaje en red para conectar zonas aisladas.

Este planteamiento se ve reforzado cuando se constata que una educación familiar coherente, exigente y rica se asocia a resultados de alto rendimiento (Jiménez, 2006). Cuando ese apoyo familiar es débil, el papel de la escuela se vuelve fundamental, ya sea siguiendo un modelo inclusivo que permita la diferenciación del currículo (Almeida y Oliveira, 2010), o mediante la aceleración.

Algunas investigaciones apuntan incluso que la educación acelerada mejora la velocidad de procesamiento de la información en alumnado superdotado, independientemente de la edad. El ambiente educativo experiencia y conocimiento- jugaría un papel importante como estímulo para incrementar el desarrollo de estos estudiantes; es decir, además de satisfacer una necesidad cognitiva, la aceleración mejora el desarrollo cognitivo (Duan, Shi y Zhou, 2010).

Otro estudio de caso, esta vez con una muestra de adolescentes vietnamitas, concluye en un más alto nivel de responsabilidad social, autocontrol y razonamiento moral entre estudiantado sobredotado frente al resto. Aunque todos alcanzaron igual puntuación en armonía y conducta ética, los de más talento se mostraron menos conservadores (Nguyen, Minh, Jin y Gross, 2013), lo que cuadra bien con una mayor capacidad creativa, como otros estudios han puesto de manifiesto (Bermejo et ál., 2010).

\section{CONECTANDO SERVICIO Y SUPERDOTACIÓN}

A lo largo del artículo se ha comentado repetidamente cómo uno de los cauces más motivadores para desarrollar el talento social es la utilidad de esas capacidades en la resolución de problemas reales (Renzulli, 2010; Renzulli et ál. 2018). Dado que el currículum no contempla sino un aprendizaje deductivo de tipo formal y prescrito, se deberían arbitrar márgenes para orientar la educación también al estudiante. El alumno puede ser protagonista si tiene no solo que buscar información, sino experimentar, analizar y preparar 
un informe acerca de alguna necesidad contextualizada, sobre todo si la personaliza para comprometerse en solucionarla. Para ello necesita enfrentarse con obstáculos, planificar la actuación, analizar opciones, argumentar con lógica, predecir consecuencias, gestionar recursos y cooperar. De ese modo podrá aprender que no existen soluciones ideales y que los problemas y respuestas afectan a personas reales, lo que genera una preocupación por la excelencia.

En este sentido, si logramos integrar el interés personal con el aprendizaje del servicio, el talento se hace altamente significativo (Bruce-Davis y Chancey, 2012). Aplicando el modelo de enriquecimiento triádico de Renzulli (Renzulli y Reis, 1997) al aprendizaje del servicio, podemos desarrollar la conciencia comunitaria en alumnado con habilidades especiales, inspirarles en el servicio a los demás y motivarles para construir un mundo mejor. El resultado podría ser una mayor facilidad para superar los obstáculos que provocan bajo rendimiento, como son la ausencia de retos, las actitudes críticas hacia la escuela, la presión negativa del grupo de iguales, o los diferentes valores percibidos, además de los problemas culturales, étnicos o de género asociados.

Ahora bien, ¿̇tiene el conjunto de adolescentes con talento un compromiso mayor por el bien común? A juzgar por algunos estudios empíricos (Bronk, Holmes y Talib, 2010) los propósitos vitales que traspasan el propio yo se despiertan en ellos antes que en sus iguales; y con una orientación más de servicio que de disfrute personal. Sin embargo, no se comprometen más que sus pares en tareas prosociales. Por tanto, se puede influir en el ambiente para llenar el vacío entre la inquietud y la acción, incidiendo en su especial responsabilidad para usar sus talentos en servir a otros. En definitiva, fomentar propósitos vitales puede ser una fuente de motivación para que los superdotados apliquen sus habilidades de modo socialmente responsable.

Su mayor propensión para la creatividad (Bermejo et ál., 2010) puede ser alentada por el profesor, de modo que toda el aula resulte beneficiada por el cultivo de la imaginación, la intuición, la curiosidad y la búsqueda de soluciones originales a problemas reales.

Los cursos específicamente dirigidos al aprendizaje del servicio, así como las prácticas de voluntariado no solo incrementan la conciencia cívica, sino que tienen incluso un positivo impacto académico, a la vez que mejora el sentido comunitario de la autoeficacia (Stewart y Bai, 2010). Si consideramos como patrimonio social (Mettrau, 2010) además de la inteligencia, cada una de las capacidades excelentes que se distribuyen de modo diverso en el estudiantado, la educación puede ponerlas al servicio de todos. El papel del profesor será clave para desarrollar capacidades interpersonales, la innovación y la renovación de las tareas y proyectos que cada uno pueda llevar a cabo en vistas del bienestar de los demás.

\section{Propuesta: la tutoría entre iguales}

El enfoque social del talento ha sido objeto de recientes investigaciones con intención de promover mayor equidad y evitar el sesgo. El panorama de los estudios sobre superdotación en España (Prieto, Sainz y Fernández, 2012; González, 2015) muestra la conveniencia de considerar además de lo cognitivo, aspectos como la personalidad, la creatividad o las competencias socioemocionales de estudiantes con habilidades especiales. La diversidad de dimensiones de la superdotación incrementa la necesidad de construir modelos adaptativos y fluidos que la afronten, entre ellos promover para estos estudiantes un liderazgo solidario en el proceso educativo. La educación centrada en el estudiante, el aprendizaje por proyectos, las comunidades prosociales pueden facilitarlo. De otra parte, la aceleración y flexibilidad de itinerarios tiene efectos sociales positivos, si bien pueden afectar a la dimensión inclusiva. Análisis como los de Tourón y Santiago (2014, 2015) y estudios empíricos como el de Pérez y Jiménez (2018) señalan acertadamente el déficit de atención a la excelencia en España. De hecho, en los últimos resultados de PISA (OCDE, 2016) se estima un 10,9\% de alumnado con nivel excelente en al menos un área de las evaluadas (matemáticas, ciencias y lengua) frente a la media de 15,3\% de los países de la OCDE. La apuesta por el modelo de Flipped Learning de estos autores busca a través de las TIC que cada estudiante personalice su aprendizaje avanzando a su propio ritmo. Nos 
parece que la deriva individualista de este enfoque podría minimizarse y aun mejorarse si se acompaña de la tutoría entre iguales como modo de adquirir competencias ético-cívicas en la propia aula.

En este sentido se han desarrollado estudios sobre el impacto social del aprendizaje de servicio, en general centrados en problemas de la comunidad.

La idea de las tutorías entre iguales entronca con la ethical mind gardneriana, recoge la dimensión social de los distintos modelos de enriquecimiento, y la diferenciación de ritmos de aprendizaje, y los concentra en una intervención marcadamente práctica de cooperación intraescolar y liderazgo flexible de los más avanzados en cada área. Sin embargo, la importancia de generar interacciones directas como actores sociales en este alumnado se enfrenta con el obstáculo de que vean su responsabilidad circunscrita solo a la familia o los amigos (Seider, 2010). De ahí que la tutoría entre iguales precise de orientación docente para dotarla de un sentido inclusivo e integrador.

También enlaza con las propuestas de aprendizaje colaborativo, facilitadas por el desarrollo de las TIC con su componente de estimulación y creatividad, en su aplicación al trabajo estudiante-estudiante. Algunas investigaciones (García-Valcárcel, Hernández y Recamán, 2012) remarcan los beneficios de este aprendizaje en grupos pequeños y homogéneos, con especial rendimiento para estudiantes desaventajados. Por otra parte, el esfuerzo de explicar a otro compañero afianza el aprendizaje propio al tener que reformularlo, a la vez que ese apoyo es mejor recibido si viene de un igual (Saura y Del Valle, 2012). A estas reflexiones cabría añadir que la tutoría de pares aumenta los beneficios sociales y de carácter, así como una mayor aceptación e igualdad, a la vez que permite solventar mejor el problema de la pasividad del receptor, puesto que la relación es personal y no de grupo.

Aunque el apoyo de los iguales ha sido objeto de atención por parte de la escuela inclusiva (The Inclusive Schools Network -ISN-), la tutoría entre iguales en este contexto amplía su alcance, al ser compatible tanto con la aceleración de materias, como con la de cursos completos, si le añadimos flexibilidad. En efecto, el apoyo mutuo entre estudiantes puede diseñarse dentro del mismo grupo aula, o bien en actividades específicas en las que estudiantes mayores orienten o dirijan el aprendizaje de alumnado del curso inferior. Cabe llevar a cabo actividades programadas dentro del plan de determinadas asignaturas en la misma clase, o bien estructurar algunas clases específicas de apoyo con alumnado sobredotado en tareas de asesoramiento a compañeros con más dificultad. En este sentido se ha propuesto que el profesor promueva para el estudiantado de más capacidad un trabajo más autónomo e independiente (Genovard, Gotzens, Badía y Dezcallar, 2010), lo cual podría intensificarse con un programa de tutorización a otros compañeros, al tiempo que adquiere orientación social e inclusiva.

La elección y el diseño del programa dependerá del área de conocimiento, del grupo-clase de que se trate, de la personalidad de los alumnos y alumnas más y menos aventajados, etc. Puede incluso considerarse la tutorización de los iguales como una tarea asignada de modo variable entre el alumnado con mejor rendimiento en cada evaluación, haciéndola depender de los logros en aprendizaje que sean capaces de suscitar en el grupo de los tutorizados. Recordemos que según PISA (Jiménez y Baeza, 2012) más que la clase social, influyen en el rendimiento las horas del currículo dedicadas a las asignaturas y al estudio, algo que las tutorías de iguales pueden reforzar tanto en el/la alumno/a-tutor/a como en el tutorado.

Un adecuado diseño y el cuidadoso seguimiento de las tareas y metas encomendadas redundará en beneficio de todos. El alumnado con algunas carencias porque verá reforzado su aprendizaje mediante la ayuda de estudiantes más aventajados. Estos, porque pondrán sus capacidades al servicio de sus iguales, reforzando su propio aprendizaje y adquiriendo conciencia de su valor social y de la trascendencia colectiva del saber. El estigma del superdotado y el posible rechazo de sus colegas (Ferrándiz et ál. 2010) puede ser combatido al ser percibido como apoyo para el progreso mutuo. La rutina y aun el aburrimiento que experimenta este alumnado pueden resultar minimizados porque la tutoría potencia la socialización con la motivación de contribuir al aprendizaje de los iguales. Incluso es posible que a través del desarrollo de estas habilidades sociales alguno de los mejores y más capacitados estudiantes descubra su futura vocación docente. 
Por último, los nuevos desarrollos del peer tutoring indican cómo no hay mejor aprendizaje para ayudar que el ser ayudado (Topping, 2015; Roscoe, Walker, y Patchan, 2018); por lo que si la atención al alumnado sobredotado se orienta a que tutoricen a su vez a otros compañeros se facilitará su propio desarrollo en virtudes sociales, así como mayor equidad e inclusión de estudiantado en desventaja. De modo que se pueden lograr altos niveles de rendimiento en todos los y las estudiantes, aunando excelencia y equidad como el último informe PISA (OCDE, 2016) sugiere a la luz de la experiencia de algunos países.

Precisamente la complejidad en la implementación de la idea deviene su principal reto para posteriores investigaciones y propuestas prácticas, pues deberán ajustarse-flexibilizarse numerosas variables, tales como el área docente, grupo-clase, perfil de la alta capacidad, diseño curricular y capacidad creativa del profesorado. Posteriores estudios de campo podrán poner en valor las intervenciones prácticas más relevantes al respecto.

\section{CoNCLUSIón}

\section{La dimensión social del desarrollo del talento. Un ejemplo: las tutorías entre iguales.}

Entre las recomendaciones que pueden desprenderse de los contenidos analizados podemos destacar las siguientes:

Sería necesario prestar especial atención a la dimensión social de las habilidades especiales del alumnado con determinados talentos, para poder darles un tratamiento adecuado a su ritmo de aprendizaje.

A ello nos impulsa la atención a su diversidad, la búsqueda de la excelencia de cada estudiante, y la mejora del conjunto, no solo del grupo aula, sino de la sociedad sostenible del futuro, que necesitará contar con todas las capacidades excepcionales que puedan aportar los jóvenes de hoy.

El aprendizaje del servicio, mediante el refuerzo de la educación social, debería jugar un papel importante en la motivación inicial del talento.

El previsible liderazgo que ejercerá el estudiantado sobredotado el día de mañana en diversos campos del saber puede y, en nuestra opinión debe, ser orientado a la resolución de problemas sociales y al bienestar colectivo, ya desde la etapa escolar.

Conviene desarrollar la mente ética del alumnado talentoso, porque amplía el foco de atención más allá de sus problemáticas individuales y de contexto inmediato, al dotarles de un sentido comunitario y orientado al futuro.

Se ha reflexionado sobre la relevancia de los aspectos cognitivos y no cognitivos de la personalidad del estudiantado, tanto para el reconocimiento de una amplia gama de habilidades excelentes -en las variadas dimensiones de la inteligencia-, como para la adecuada orientación de su ejercicio. Pues bien, una forma de fomentar el desarrollo de esas capacidades, y a la vez de evitar la excepcionalidad comparativa y a veces segregadora de su manifestación, consiste en compartirlas, poniéndolas al servicio de los compañeros.

La educación social debería permear transversalmente los aprendizajes escolares para promover, practicar y asumir una enseñanza cívica, prosocial y democrática, una actitud positiva y comprensiva hacia los demás, la responsabilidad por el otro y el liderazgo constructivo; a la vez que construye la conciencia ética de que adolecen a veces los líderes sociales.

La educación social debería permear transversalmente los aprendizajes escolares para promover, practicar y asumir una enseñanza cívica, prosocial y democrática, una actitud positiva y comprensiva hacia los demás, la responsabilidad por el otro y el liderazgo constructivo; a la vez que construye la conciencia ética de que adolecen a veces los líderes sociales.

Es en el ámbito escolar donde más fácilmente encontramos los problemas reales, los conflictos que requieren atención práctica, las interacciones afectadas por los diversos tipos de discriminación, entre ellos las diversas formas de talento. 
Fomentar las tutorías entre iguales pueden aportar en cada materia de enseñanza un apoyo para todo el alumnado; no solo porque el estudiantado talentoso contribuirá a la mejora de sus iguales, sino porque también desarrollará sus habilidades sociales y, al ponerlas al servicio de los demás, crecerán ellos mismos como personas.

\section{ReFERENCIAS}

Aljughaiman, A. (2010). El Oasis: un modelo de enriquecimiento para el desarrollo del talento. Revista electrónica interuniversitaria de formación del profesorado, 13(1), 75-84.

Almeida, L. S. y Oliveira, E. (2010). Los alumnos con características de sobredotación: la situación actual en Portugal. Revista electrónica interuniversitaria de formación del profesorado, 13(1), 85-95.

Assouline, S. G., Colangelo, N., Lupkowski-Shoplik, A., Lipscomb, J. y Forstadt, L. (2009). Iowa acceleration scale manual: a guide for whole-grade acceleration. Scottsdale, AZ: Great Potential Press.

Bermejo, R., Hernández, D., Ferrando, M., Soto, G., Sainz, M., y Prieto, M.D. (2010). Creatividad, inteligencia sintética y alta habilidad. Revista electrónica interuniversitaria de formación del profesorado, 13(1), 97-109.

Blumen, S. (2008). Motivación, sobredotación y talento: un desafío para el éxito. Revista de Psicología, 26(1), 147-184.

Blumen, S. (2013). New trends in talent development in Peru. Journal for the Education of the Gifted, July 2, 346-364. http://dx.doi.org/10.1177/0162353213492925

Bronk, K.C., Holmes, W., y Talib, T.L. (2010). Purpose in life among high ability adolescents. High Ability Studies, 21(2), 133-145. http://doi.org/10.1080/13598139.2010.525339

Bruce-Davis, M.N., y Chancey, J.M. (2012). Connecting students to the real world: developing gifted behaviors through service learning. Psychology in the Schools, 49(7), 716-723. http://doi.org/10.1002/pits.21622

Castelló A. (2002). Curso sobre detección, identificación e intervención de alumnado de Altas Capacidades. Gran Canaria: Dirección General de Ordenación e Innovación Educativa de Canarias.

Colangelo, N., Assouline, S. G. y Gross, M. U. (2004). A nation deceived: how schools hold back America's brightest students. Iowa City: The Belin Blank International Center for Gifted Education and Talent Development.

Cunha F. y Heckman, J. J. (2010). Investing in Our Young People. En A. J. Reynolds, A.J. Rolnick, M. M. Englund, y. A. Temple (Eds.), Childhood programs and practices in the first decade of life (pp. 381-414). New York: Cambridge University Press.

Duan, X., Shi, J. y Zhou, D. (2010). Developmental Changes in Processing Speed: Influence of Accelerated Education for Gifted Children, Gifted Child Quarterly 54(2) 85-91. http://doi.org/10.1177/0016986209355971

Ferrándiz, C., Prieto, M.D., Fernández, M.C., Soto, G., Ferrando, M. y Badía, M.M. (2010). Modelo de identificación de alumnado con altas habilidades de Educación Secundaria. Revista Electrónica Interuniversitaria de Formación del Profesorado, 13 (1), 63-74

Gagné, F. (2015). From genes to talent: the DMGT/CMTD perspective, Revista de Educación, 368, 12-37. http://d oi.org/10.4438/1988-592X-RE.2015-368-289

Gallagher, H., y Gallagher, S. (1994). Teaching the gifted child. Boston: Allyn y Bacon.

Gallagher, J. (2004). Public policy and acceleration of gifted students. En N. Colangelo, S. Assouline y M. Gross (Eds.), A nation deceived: how schools hold back America's brightest students (pp. 39-46). Iowa City: The Belin Blank Center Gifted Education and Talent Development.

García, A., Hernández, A. y Recamán, A. (2012). La metodología del aprendizaje colaborativo a través de las TIC: una aproximación a las opiniones de profesores y alumnos. Revista Complutense de Educación, 23(1), 161-188. http ://dx.doi.org/10.5209/rev_RCED.2012.v23.n1.39108

Gardner, H. (2008). The five minds for the future. Schools, 5(1-2), 17-24.

Genovard, C., Gotzens, C., Badía, M.M. y Dezcallar, M.T. (2010). Los profesores de alumnos con altas habilidades. Revista Electrónica Interuniversitaria de Formación del Profesorado, 13(1), 21-31.. 
González, M. (2015). Perfiles cognitivos asociados a alumnos con altas capacidades intelectuales (Tesis doctoral). Universidad de Alicante, Alicante. Recuperado de https://rua.ua.es/dspace/bitstream/10045/50251/1/tesis_g onzalez_garcia.pdf

Gradstein, M. y Justman, M. (2002). Education, social cohesion, y economic growth. American Economic Review, 92(4), 1192-1204.

Hernández, D., Ferrándiz, C., Ferrando, M., Prieto L. y Fernández, M.C. (2014). The theory of multiple intelligences in the identification of high-ability students. Anales de psicologia, 30(1), 192-200. http://dx.doi.org/10.6018/a nalesps.30.1.148271

Hernández, D. y Gulsah, A. (2014). A cross-cultural perspective about the implementation and adaptation process of the schoolwide enrichment model. The importance of talent development in a global world. Gifted Education International, 14, 1-14. http://doi.org/10.1177/0261429414526335

Inkeles, A. (1966). Social structure and the socialization of competence. Harvard Educational Review, 36(3), 265-283. doi: http://dx.doi.org/10.17763/haer.36.3.00415586k1121763

Inkeles, A., y Smith, D. (1974). Becoming Modern: Individual Changes in Six Developing Societies. Cambridge, MA: Harvard University Press.

Jiménez, C. (2006). Educación familiar y alumnos con alto rendimiento. Revista Española de Pedagogía, 234, 273-300.

Jiménez, C., y Baeza, M.A. (2012). Factores significativos del rendimiento excelente: PISA y otros estudios. Ensaio: Avaliação e Políticas Públicas em Educação, 20(77), 647-676.

LeCompte, K., y Blevins, B. (2013). New horizons in civics education. En W.B. Russell (Ed.), Digital Social Studies (pp. 345-369). Charlotte, NC: Information Age Publishing.

Levin, H.M. (2013). The utility and need for incorporating noncognitive skills into large-scale educational assessments. En M. Davier, E. González, I. Kirsch y K. Yamamoto. (Eds.), The role of international large-scale assessments: perspectives from technology, economy, and educational research (pp. 67-86). Netherlands: Springer.

Levinson, M. (2012). No citizen left behind. Cambridge, MA: Harvard University Press.

Llamas, F. y Macías, E. (2016). Formación inicial de docentes en educación básica para la generación de conocimiento con las Tecnologías de la Información y la Comunicación. Revista Complutense de Educación, 29(2), 577-593. h ttp://dx.doi.org/10.5209/RCED.53520

López, L. y Calero, M.D. (2018). Sobredotación, talento e inteligencia normal: diferencias en funciones ejecutivas, potencial de aprendizaje, estilo cognitivo y habilidades interpersonales. Revista de Educación Inclusiva, 11(1), 91-112.

López, V. (2007). La Inteligencia Social: Aportes Desde su Estudio en Niños y Adolescentes con Altas Capacidades Cognitivas. Psykhe, 16(2), 17-28. http://doi.org/10.4067/S0718-222820070002000255

Mettrau, M.B. (2010). Programas académicos para la formación de profesores de alumnos con superdotación y talento. Revista electrónica interuniversitaria de formación del profesorado, 13(1), 159-167.

Mohammadyari, G., (2013). Emotional intelligence as predictor of academic achievement among gifted students. Technical Journal of Engineering and Applied Sciences, 3(15), 1560-1563.

Mora, O., Mathiesen, M.E., Merino, J.M., Castro, G. y Navarro, G. (2010). Desarrollo socioemocional y rendimiento académico en alumnos de alto potencial intelectual. Chile: Universidad de Concepción. Recuperado de https://s cholar.google.com/scholar?oi=bibsyhl=esycluster $=4109644708509350256$

Nguyen, P., Minh, T., Jin, P. y Gross, M. U. (2013). Confucian Values in Vietnamese Gifted Adolescents and their Non-Gifted Peers. Gifted and Talented International, 28(1), 227-238. http://dx.doi.org/10.1080/15332276.2 013.11678417

OCDE (2016). PISA 2015. Resultados Clave. Recuperado de https://www.oecd.org/pisa/pisa-2015-results-in-focus -ESP.pdf

Olzewski, P., Subotnik, R., y Worrell, F. (2015). Rethinking Giftedness: A Developmental Approach. Revista de Educación, 368, 245-267. http://doi.oeg/10.4438/1988-592X-RE-2015-368-297 
Peñas, M. (2008). Caracteristicas socioemocionales de las personas adolescentes superdotadas. Ajuste psicológico y negación de la superdotación en el concepto de sí mismas. Madrid: CIDE, Secretaría General Técnica, Ministerio de Educación, Política social y deporte.

Pérez, J. (2005). Repensar la familia. Madrid: Ediciones Internacionales Universitarias.

Pérez, L. y Jiménez, C. (2018) Influencia de la organización escolar en la educación de los alumnos de altas capacidades. Enseñanza y Teaching, 36(1), 151-178. https://doi.org/10.14201/et2018361151178

Pfeiffer, S. (2015). Tripartite Model of Giftedness and Best Practices in Gifted Assessment. Revista de Educación, 368, 156-182. http://doi.org/10.4438/1988-592X-RE-2015-368-293

Plucker, J. A. y Callahan, C. M. (2014). Research on Giftedness and Gifted Education: Status of the Field and Considerations for the Future. Exceptional Children, 80(4), 390-406. http://doi.org/10.1177/001440291452 7244

Prieto, L., Sainz, M. y Fernández, M. C. (2012). Estudio de la superdotación en España. AMAzônica, 5, 10(3), 48-78, (Extra).

Prieto, M.D. (2004). Evaluación de talentos específicos desde el modelo de las inteligencias múltiples. En C. Jiménez y A. Rivero. (Eds.), Diagnóstico y atención a los alumnos con necesidades educativas especificas: alumnos intelectualmente superdotados (pp. 45-66). Madrid: MEC, Subdirección general de Información y Publicaciones.

Renzulli, J. S. (2010). El rol del profesor en el desarrollo del talento. Revista electrónica interuniversitaria de formación delprofesorado, 13(1), 33-40.

Renzulli, J. S. (2012). Reexamining the role of gifted education and talent development for the 21 st Century: a fourpart theoretical approach. Gifted Child Quarterly, 56(3), 150-159. http://doi.org/10.1177/0016986212444901

Renzulli, J. S. y D'Souza, S. (2014). Intelligences outside the normal curve: Co-cognitive factors that contribute to the creation of social capital and leadership skills in young people. En J.A Plucker y C.M Callahan. (Eds.), Critical issues and practices in gifted education: What the research says (pp. 343-362). Waco TX: Prufrock Press.

Renzulli, J.S., y Reis, S. M. (1997). The schoolwide enrichment model: A how-to guidefor educational excellence. Mansfield Center, CT: Creative Learning Press.

Renzulli, J.S., Sands, M.M. y Heilbronnor, N.N. (2018). Operation houndstooth: a positive perspective on developing social intelligence. Sobredotaçao 15(2), 209-243.

Robinson, N. (2004). Effects of academic acceleration on the social-emotional status of gifted students. En N. Colangelo, S. G. Assouline y M. U. Gross. (Eds.), A nation deceived: how schools hold back America's brightest students (pp. 59-67). Iowa City, IA: The Belin Blank Center Gifted Education and Talent Development.

Rogers, K. (2007). Lessons learned about educating the gifted and talented: a synthesis of the research on educational practice. Gifted Child Quarterly, 51(4), 382-396. http://doi.org/10.1177/0016986207306324

Roscoe, R.D., Walker, E.A., y Patchan, M.M. (2018). Facilitating peer tutoring and assessment in intelligent learning systems. In S D. Craig (Ed.), Tutoring and Intelligent Tutoring Systems (pp. 41-68). New York: Nova Science Publishers, Inc

Saura, D., y Del Valle, J. (2012). Implantación del modelo de aprendizaje cooperativo en Secundaria Obligatoria. En A. Hernández y S. Olmos. (Eds.), Metodologias de aprendizaje colaborativo a través de las tecnologias (pp. 95-105). Salamanca: Aquilafuente.

Seider, S. (2010). Developing the Ethical Minds of Gifted Adolescents. Gifted Education International, 27 (2) 132-148. http://doi.org/10.1177/026142941002700204.

Sternberg, R.J. (2018). 21 Ideas: A 42-Year Search to Understand the Nature of Giftedness. Roeper Review, 40(1), 7-20. https://doi.org/10.1146/annurev-psych-010418-102846

Sternberg, R.J., Grigorenko, E.L., Prieto, M.F., Hernández, D., García, C.F., García, M.R.B., y Sánchez, M.D.P. (2010). Enseñanza de la inteligencia exitosa para alumnos superdotados y talentosos. Revista electrónica interuniversitaria de formación del profesorado, 13(1), 111-118. 
Stewart, T., y Bai, H. (2010). Community service self-efficacy and summer service-learning: comparative analyses among academically talented youth. Gifted Education International, 27(2), 149-160. http://doi.org/10.1177/ 026142941002700205

Topping, K. (2015). Peer tutoring: old method, new developments. Journal for the Study of Education and Development, 38 (1), 1-29, http://dx.doi.org/10.1080/02103702.2014.996407

Tourón, J. (2010) El desarrollo del talento y la promoción de la excelencia: exigencias de un sistema educativo mejor. Bordón. Revista de pedagogía, 62(3), 133-149.

Tourón, J., y Santiago, R. (2014). Talent Development in a Digital Era. Symposium. 14th Conference of the European Council for High Ability. Ljubljana (Slovenia).

Tourón, J., y Santiago, R. (2015). El modelo Flipped Learning y el desarrollo del talento en la escuela. Revista de Educación, 368, 196-231. http://doi.org/10.4438/1988-592X-RE-2015-368-288

Tourón, J., y Tourón, M. (2016). Identification of verbal and mathematical talent: the relevance of "out of level" measurement. Anales de psicologia, 32(3), 638-651 http://doi.org/10.6018/analesps.32.3.259401

Tyler, F. B. (2004). The role of prosocial communities in youth development. Psykhe, 13(2), 3-15. http://doi.org/10 $.4067 / \mathrm{S} 0718-222820040002000170$

Valadez, M. D., Pérez, L., y Beltrán, J. (2010). La inteligencia emocional de los adolescentes talentosos. Faisca: revista de altas capacidades, 15(17), 2-17.

Van der Westhuizen, C. N., y Maree, J. G. (2010). From human to humankind: Facilitating global awareness among the gifted. Gifted Education International, 27(1), 54-62. http://doi.org/:10.1177/026142941002700110

West, D. (2012) How blogs, social media, and video games improve education. Washington, D.C.: Brookings Institute Governance Studies.

CC BY-NC-ND 